\title{
Alveolar rhabdomyosarcoma infiltrating bone marrow at presentation: the value to diagnosis of bone marrow trephine biopsy specimens
}

\author{
M M Reid, P W G Saunders, N Bown, C R Bradford, Z T Maung, A W Craft, \\ A J Malcolm
}

\begin{abstract}
Aims: To describe the histological appearances of bone marrow infiltrated with rhabdomyosarcoma at presentation and to determine their value in establishing the diagnosis.

Methods: Patients presenting over seven years in the northern health region of England with rhabdomysarcoma were studied. Bone marrow aspirates and trephine biopsy specimens taken at presentation were examined.

Results: Seven of 32 patients with rhabdomyosarcoma had bone marrow infiltration, resulting in marrow failure in all cases, at diagnosis. The diagnosis was established in these seven by the typical cytological appearances and immunophenotype of the infiltrating cells (all seven patients) and cytogenetic abnormalities (three patients). Histological examination of the bone marrow showed a pseudoalveolar pattern with fibrous septal bands, enlarged vascular channels, and lack of cohesion of the tumour cells within the subdivided aggregates in all seven. In four cases multinucleate giant cells, often with peripherally sited nuclei, were found.
\end{abstract}

Conclusions: These histological features of infiltrated marrow are so characteristic that the diagnosis of alveolar rhabdomyosarcoma can be made, or at least suspected, in many cases even without recourse to technically difficult and expensive further investigations. Bone marrow biopsy should be a routine part of the investigation of patients with bone marrow failure and will be of particular value in the diagnosis of those with disseminated alveolar rhabdomyosarcoma.

Rhabdomysarcoma, the commonest soft tissue sarcoma of children and adolescents, may be widely disseminated at the time of presentation. Etcubanas et al ${ }^{1}$ reported a series of 10 cases in whom extensive search failed to reveal the primary tumour. They and others ${ }^{23}$ emphasised the difficulty of making the diagnosis in such cases. They described in some detail the cytological features of the infiltrating cells but made no mention of the bone marrow histology.

We present details of the bone marrow appearances of all cases of rhabdomyosarcoma with marrow infiltration at presentation recognised in the northern health region of England between January 1985 and December 1991, and highlight the characteristic histological features of infiltrated bone marrow.

\section{Methods}

Most cases of childhood cancer in the northern health region of England (total population 3.08 million) are referred to hospitals in Newcastle upon Tyne for treatment. The Northern Region Children's and Young Person's Malignant Disease Registry collects data on all cases aged less than 25 years at diagnosis. Finally, haematologists within the region communicate informally and exchange information about malignant infiltration of bone marrow. All three sources were used to obtain and crosscheck data on cases of rhabdomyosarcoma for this retrospective study.

In all cases without bone marrow failure and with an obvious primary tumour the diagnosis was made by standard histological and immunohistochemical methods from a biopsy specimen of the primary tumour. Tumour histology was reviewed by one of the authors (AJM). Bilateral iliac crest bone marrow aspirate and biopsy are routine parts of initial staging procedures in all children aged under 15 years but are not always carried out in older patients. In patients with bone marrow infiltration, all of whom had bone marrow failure in this series, the diagnosis was made by bone marrow examination alone.

Romanovsky stained marrow smears, supplemented by other cytochemical stains, and sections of bone marrow biopsy specimens stained with haematoxylin and eosin, and silver for reticulin, were examined. Various standard immunocytochemical or immunohistochemical stains were performed on the smears and biopsy specimens in the hospitals where the bone marrow samples were obtained. Among these the monoclonal antibodies UJ13A, UJ127.11, and various antisera against myoglobin, desmin, actin, vimentin and common leucocyte antigen were those most commonly used. All bone marrow specimens were reviewed by one of the authors (MMR).

Cytogenetic analysis of bone marrow derived metaphases was carried out using standard G-banding techniques.

\footnotetext{
Correspondence to: Dr M M Reid

Accepted for publication 14 February 1992

Division of Pathology, Sciences, University of Newcastle upon Tyne
} 


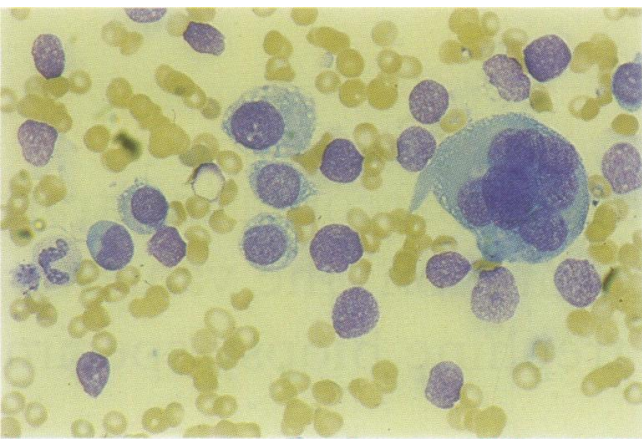

Fig 1

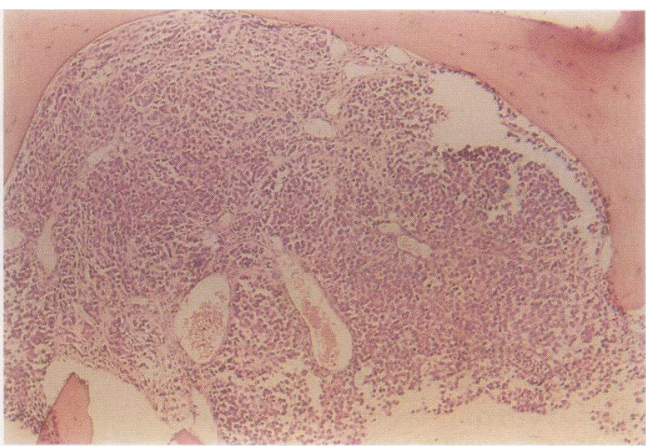

Fig 3

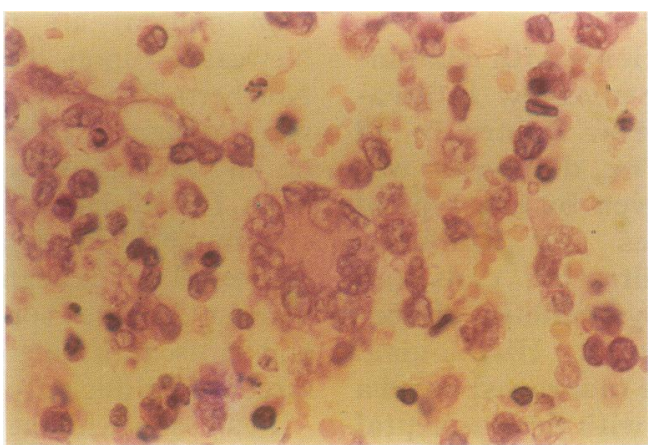

Fig 5

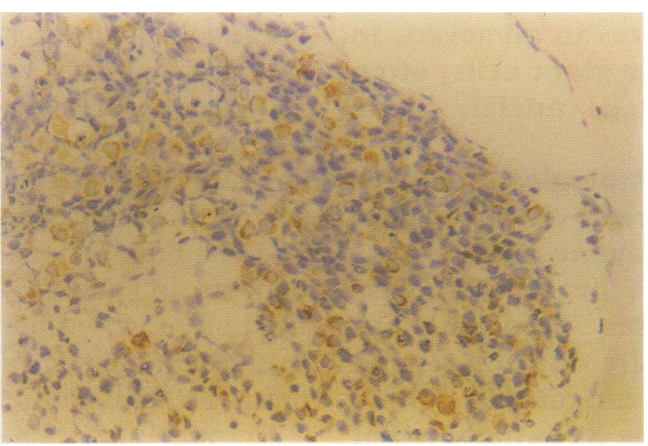

Fig 7

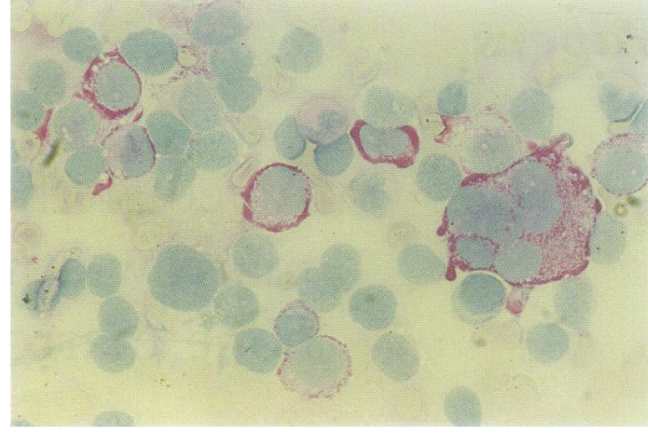

Fig 2

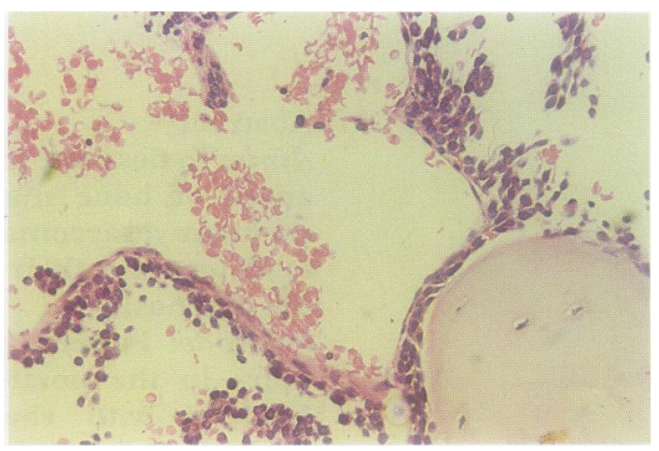

Fig 4

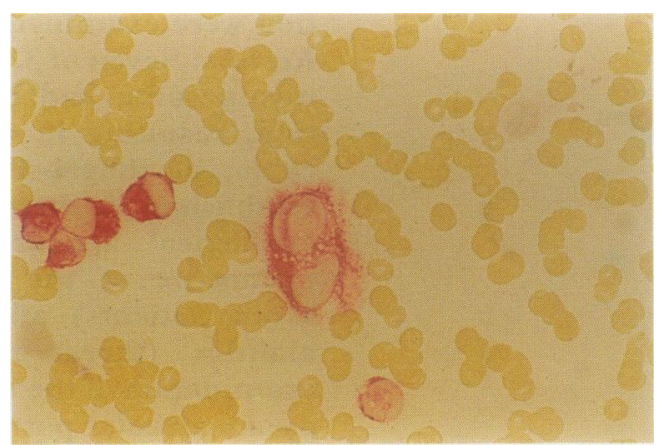

Fig 6

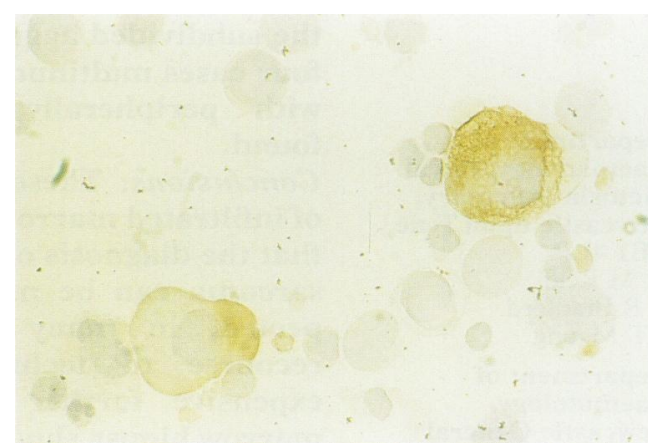

Fig 8

Figure 1 Bone marrow aspirate. Primitive cells with cytoplasmic vacuolation (Leishman).

Figure 2 Bone marrow aspirate. The distribution of lakes of PAS positive material is similar to that of the vacuoles in figure 1.

Figure 3 Bone marrow trephine biopsy specimen. A relatively solid area of tumour, intersected by fibrous septa, with some dilated vascular channels (haematoxylin and eosin).

Figure 4 Bone marrow trephine biopsy specimen. Tumour cells adhering to the margins of a vascular channel but with loss of central cohesion (haematoxylin and eosin).

Figure 5 Bone marow trephine biopsy specimen. Multinucleate giant cell with peripherally sited nuclei (haematoxylin and eosin).

Figure 6 Bone marrow aspirate. Immunocytological demonstration of desmin. Most cells were strikingly positive (immunoalkaline phosphatase).

Figure 7 Bone marrow trephine biopsy specimen from the patient in figure 6. Immunohistological demonstration of desmin is less striking (immunoperoxidase).

Figure 8 Bone marrow aspirate. Immunocytological demonstration of myoglobin (immunoperoxidase). 
Immunophenotype and cytogenetic abnormalities of bone marrow tumour cells

\begin{tabular}{|c|c|c|c|c|c|c|c|c|}
\hline Case No & Myoglobin & Desmin & Actin & Vimentin & $\begin{array}{l}\text { Common } \\
\text { leucocyte } \\
\text { antigen }\end{array}$ & $U f 13 A$ & Uf127.11 & Cytogenetic findings \\
\hline 1 & - & + & + & + & - & - & - & $2 q+$ \\
\hline 2 & + & + & + & + & - & + & - & failed \\
\hline 3 & - & + & ND & + & - & ND & ND & $2 q+$ \\
\hline 4 & ND & + & ND & + & - & ND & ND & failed \\
\hline 5 & - & + & + & ND & ND & + & - & $\mathrm{t}(2 ; 13), 1 \mathrm{q}+, \mathrm{i}(18 \mathrm{q})$ \\
\hline 6 & - & + & ND & ND & ND & ND & ND & ND \\
\hline 7 & - & + & + & ND & ND & + & - & $t(14 ; 20)$ \\
\hline
\end{tabular}

\section{Results}

During the seven year period 20 cases of rhabdomyosarcoma were diagnosed in children under 15 years old. All had a bone marrow examination. Three had infiltrated marrow at presentation. Eleven cases aged 15 to 24 years were identified, six of whom had a bone marrow examination. Three had infiltrated marrow. One 32 year old man also presented during the study period with widespread rhabdomyosarcoma involving the bone marrow. All seven with infiltrated marrow but none of the remaining 25 had bone marrow failure. The older man also had disseminated intravascular coagulation. The primary tumours in these seven were paraspinal $(n=2)$, forearm $(n=1)$, calf $(n=1)$, pelvic $(n=1)$, base of skull $(n=1)$ and unknown $(\mathrm{n}=1)$.

\section{BONE MARROW CYTOLOGY}

All seven had heavy infiltrates of malignant cells. In all there was some tendency for the cells to clump, though not as strikingly as in neuroblastoma. Multinuclearity of malignant cells was found in all cases. In some only occasional multinucleate cells with up to three nuclei per cell were seen, but in others some giant cells with over 10 nuclei were also present. All cases showed vacuolation of the cytoplasm in at least some cells. Vacuoles were often strikingly large, and coalesced into peripherally located, elongated lakes. Periodic acid Schiff positivity was present in some or most of the cells in all cases. Its location seemed to coincide with the vacuoles in most cells, but in a few was uniformly distributed throughout. In five of the seven cases phagocytosis of cellular material by occasional tumour cells was seen. In four cases the ingested material consisted of red cells or erythroblasts. In one, unidentified nucleated cells had been ingested. Examples of the cytological features are shown in figs 1 and 2.

HISTOLOGICAL FEATURES

The infiltrate was never a monotonous sheet of cells as seen in acute leukaemia. In all cases it was intersected by fibrous septal bands and contained many dilated vascular channels. Tumour cells tended to adhere firmly to the septal bands and to the margins of the vascular channels, but lacked cohesion in the centre of the subdivided aggregates. Occasional multinucleate giant cells, sometimes with peripherally sited nuclei, were found in four of the seven cases. Reticulin was noticeably increased in the septal bands and around the vascular channels, but only modestly and variably within the aggregates of tumour cells. Examples of the histological features are shown in figs 3,4 , and 5 .

IMMUNOPHENOTYING

In general the results of immunostaining were more striking in the cytological than histological preparations. In one patient desmin positive and in another desmin and actin positive tumour cells were seen in the biopsy specimen but not in the aspirated material, perhaps a result of technically inadequate preparations. The results of the most commonly used immunodiagnostic tests are shown in the table. Examples of immunopositive cells in smears and sections of marrow are shown in figs 6,7 , and 8 .

\section{CYTOGENETIC ANALYSIS}

Bone marrow cells from six patients were cultured. Two failed. Four had clonal abnormalities, with $2 \mathrm{q}+$ in two and $t(2 ; 13)$ (q36;q14), among other structural abnormalities, in the 32 year old man. One case had $14 q^{-}, 20 q+$, probably representing a translocation between these two chromosomes, but the breakpoints could not be identified. The results are shown together with the immunophenotypic data in the table.

\section{Discussion}

This series of seven cases of bone marrow infiltration with alveolar rhabdomyosarcoma represents all recognised cases occurring in a seven year period in a single health region. Those aged under 15 years at diagnosis arose in a complete and unselected series of children with rhabdomyosarcoma. For those aged 15 years or older the figures are less certain. Registry data for this age group are not yet complete, there may be bias towards earlier identification of those with bone marrow failure, not all older patients have had bone marrow examinations and, lastly, disseminated rhabdomyosarcoma may have been misdiagnosed as some other non-haemopoietic malignancy. None the less the results emphasise the overall rarity of cases of rhabdomyosarcoma with infiltrated marrow, illustrating the lack of opportunity for pathologists or haematologists to become familiar with the picture. There are too few cases here to permit useful comment on the prevalence of marrow infiltration at presentation. However, the main purpose of this report is to describe the histological appearances, rather than the prevalence, of infiltrated marrow in all recognised cases. As Etcubanas et al have shown, misdiagnosis of alveolar rhabdomyosarcoma is common. ${ }^{1}$ Until this is remedied, studies of the prevalence of bone marrow pathology in this disease will remain flawed. 
Because none of these seven cases with bone marrow disease had a formal histological diagnosis made from a biopsy specimen of the primary tumour, it is important to assess the confidence with which the diagnoses were made. In three the tentative diagnosis of rhabdomyosarcoma was put forward after seeing the cytological appearances alone. Any doubts as to whether these malignant cells might be of haemopoietic origin were dispelled, in all cases, on first sight of bone marrow histology. The appearances did not resemble leukaemia or lymphoma.

Further confirmation of the non-haemopoietic nature of the malignant cells came from the immunological studies. Various combinations of N-CAM (detected by UJ13A), actin, desmin, and vimentin positivity and common leukocyte negativity excluded haemopoietic malignancy, and were compatible with rhabdomyosarcoma. ${ }^{4}$ Desmin was detected in all seven cases. In case 2 myoglobin was convincingly shown. When taken together with the characteristic cytological features, ${ }^{12}$ these results pointed strongly to a diagnosis of rhabdomyosarcoma.

In three cases $(1,3$, and 5$)$ cytogenetic data further strengthened this view. No case had cytogenetic abnormalities suggestive of Ewing's sarcoma, peripheral neuroectodermal tumour, or neuroblastoma. The characteristic translocation $\mathrm{t}(2 ; 13)$ has been used by others to confirm a diagnosis of rhabdomyosarcoma in tissue from both primary tumour ${ }^{5}$ and infiltrated bone marrow. ${ }^{3}$ In case 5 the complete translocation was found. In cases 1 and 3 the $2 \mathrm{q}+$ marker chromosome was clearly identified, although the affected chromosome 13 was not. Case 7 had a probable t(14q;20q), previously unrecognised in rhabdomyosarcoma but not characteristic of any other malignancy.

Finally, the pseudo-alveolar pattern found in the bone marrow biopsy specimens of all seven patients closely resembles that of primary alveolar rhabdomyosarcomas. ${ }^{6}$ The combination of the cytological, immunological, cytogenetic and histological features led us to conclude that all seven cases had disseminated alveolar rhabdomyosarcoma. Although the metastatic tumour in all the cases reported here had alveolar features, it is clear that other histological variants of rhabdomyosarcoma can metastasise to bone marrow. For example, Etcubanas et al made firm diagnoses of mixed alveolar/embryonal tumour in four, undifferentiated in two and embryonal in one of their series of 10 cases with bone marrow infiltration. ${ }^{1}$ The distinction between the various types of rhabdomyosarcoma is not always clear cut. ${ }^{6}$ Those with any alveolar component may be more likely to metastasise to bone marrow, and in such cases the bone marrow metastases will usually have an alveolar pattern.

There are no details of the histological appearances of bone marrow in this disease in most standard histopathology texts, although references to the alveolar pattern of metastases in other tissues can be found. ${ }^{6}$ Few haematologists are familiar with this histological picture and it is difficult to find adequate descriptions in the haematological literature. For example, although two atlases ${ }^{78}$ and a monograph on bone marrow pathology ${ }^{9}$ contain illustrations, there is little accompanying description. The features reported by Etcubanas et al are restricted to bone marrow aspirate smears. ${ }^{1}$ Indeed, in only two of their 10 cases was the bone marrow biopsied and in neither did they describe the histological picture. Perhaps the lack of information in standard texts has been perpetuated by reluctance on the part of oncologists to request a biopsy as part of the bone marrow examination. This vicious circle has been broken in the case of neuroblastoma ${ }^{10}$ and should be in this and other cancers in which dissemination to bone marrow is known to occur.

In summary, the histological features of bone marrow infiltrated by alveolar rhabdomyosarcoma at presentation are, in our experience, so characteristic that the correct diagnosis can often be made before the results of other technically difficult and expensive tests are available. The histological appearances could raise the level of suspicion in the minds of pathologists or haematologists unused to this disease and bring about more focused, further investigation. This might be of particular benefit in patients whose primary tumour is sited where it can not easily or safely be biopsied as well as in those in which it can not be found. This series confirms the potential value of cytogenetic investigations in the diagnosis of marrow infiltration by non-haemopoietic tumour. However, because even a combination of cytogenetic and immunological approaches may, in some cases, fail to provide conclusive evidence, bone marrow biopsy of at least one iliac crest should be a routine part of the investigation/staging of patients with bone marrow failure and will be of diagnostic value in those with disseminated alveolar rhabdomyosarcoma.

We thank Mrs L More from the Northern Region Children's and Young Person's Malignant Disease Registry for help with and Young Person's Malignant Disease Registry for help with case ascertainment, Dr J T Kemshead for donating UJ13A and
UJ127.11, and Dr R Finney for allowing us to review the bone marrow specimens from one of the patients.

1 Etcubanas E, Peiper S, Stass S, Green A. Rhabdomyosarcoma, presenting as disseminated malignancy from an sarcoma, presenting as disseminated malignancy from an unknown primary site: a retrospective study of
pediatric cases. Med Pediatr Oncol 1989;17:39-44.

pediatric cases. Med Pediatr Oncol 1989;17:39-44.
2 Henderson DW, Raven JL, Pollard JA, Walters M N-I. Bone marrow metastases in disseminated alveolar rhabdomyosarcoma: a case report with ultrastructural study and review. Pathology 1976;8:329-41.

3 Brichard B, Ninane J, Gosseye S, et al. A disseminated alveolar rhabdomyosarcoma in a 9 year old boy disclosed by chromosomal translocation $(2 ; 13)(\mathrm{q} 35 ; \mathrm{q} 14)$. Pediatr Hematol Oncol 1991;8:215-9.

4 Kemshead JT, Clayton J, Patel K. Monoclonal antibodies used for the diagnosis of the small round cell tumours of childhood. In: Kemshead JT, ed. Pediatric tumors: Immunologic and molecular markers. Boca Raton Florida: CRC Press Inc, 1989:31-45.

5 Fletcher JA, Kozakewich HP, Hoffer FA, et al. Diagnostic relevance of clonal cytogenetic aberrations in malignant soft tissue tumors. N Engl $₹$ Med 1991;324:436-42.

6 Enzinger FM, Weiss SM. Soft tissue tumors. Philadelphia: C V Mosby Company, 1988:448-88.

7 Hann IM, Lake BD, Pritchard J, Lilleyman J. Colour atlas of paediatric haematology. Oxford: Oxford University Press, 1990:99.

8 Frisch B, Bartl R. Atlas of bone marrow pathology. Dordrecht: Kluwer Academic Publishers, 1990:197.

9 Wittels B. Surgical pathology of bone marrow. Philadelphia: W B Saunders Company, 1985:152-5.

10 Brodeur GM, Seeger RC, Barrett A, et al. International criteria for diagnosis, staging and response to treatment in patients with neuroblastoma. $\mathcal{f}$ Clin Oncol 1990;6:1874-81. 